\title{
Trefoil factor family 2 expression inhibits gastric cancer cell growth and invasion in vitro via interactions with the transcription factor $\mathrm{Sp3}$
}

\author{
YILING CAI, MENGTING YI, DAJUN CHEN, JINGJING LIU, BAYASI GULENG, JIANLIN REN and HUAXIU SHI \\ Department of Gastroenterology, Zhongshan Hospital Affiliated to Xiamen University, Xiamen, Fujian 361004, P.R. China
}

Received August 6, 2015; Accepted September 9, 2016

DOI: $10.3892 /$ ijmm.2016.2739

\begin{abstract}
The trefoil factor family (TFF) is a group of short secretory peptides of gastric mucous neck cells. The loss of TFF2 protein expression enhances gastric inflammation and occurs in gastric cancer. In this study, we examined the effect of TFF2 on gastric cancer cell lines in vitro and characterized the interaction between TFF2 and Sp3, including the mechanisms that mediate this interaction, using genomics and proteomics approaches, as well as genetics techniques, such as RNA interference and gene knockdown. Assays were performed to examine the role of TFF2 and Sp3 in cancer cell proliferation, invasion and migration. We found that TFF2 expression inhibited the proliferation and invasion capacity of gastric cancer cells, and induced apoptosis. TFF2 interacted with the Sp3 protein, as shown by immunofluorescence staining and immunoprecipitation with western blot analysis. Sp3 knockdown in gastric cancer cells antagonized TFF2 antitumor activity. Additionally, TFF2 upregulated the expression of pro-apoptotic proteins, such as Bid, but downregulated the expression of NF- $\kappa \mathrm{B}$ and the anti-apoptotic proteins, Bcl-xL and Mcl-1. By contrast, Sp3 knockdown significantly blocked TFF2 activity, affecting the expression of these proteins. The data from our study demonstrate that the antitumor activity of TFF2 is mediated by an interaction with the Sp3 protein in gastric cancer cells. Additional in vivo and ex vivo warrned in order to fully characterize this interaction.
\end{abstract}

\section{Introduction}

Gastric cancer is one of the most common malignancies worldwide, and is the second leading cause of cancer-related mortality; it causes $>730,000$ cancer-related deaths annually $(1,2)$. More than $70 \%$ of new cases and deaths occur in developing coun-

Correspondence to: Dr Huaxiu Shi, Department of Gastroenterology, Zhongshan Hospital Affiliated to Xiamen University, 201 Hubin Road, Xiamen, Fujian 361004, P.R. China

E-mail: huaxiushi@xmu.edu.cn

Key words: trefoil factor family 2, gastric cancer, invasion, transcription factor, $\mathrm{Sp} 3$ tries, particularly in Eastern Asia, Eastern Europe and South America (1); gastric cancer rates have decreased substantially in most parts of the world (3). Most cases are caused by Helicobacter pylori (H. pylori) infection, but tobacco smoking and dietary factors, such as intake of smoked foods, salted fish and meat, and pickled vegetables, have also been associated with a risk for gastric cancer development (1). To date, surgery is the most common treatment option for gastric cancer, followed by chemotherapy and radiation therapy, or a combination of both. However, surgical interventions are curative in $<40 \%$ of cases as gastric cancer patients are typically diagnosed at advanced stages. Thus, in order to improve gastric cancer survival and reduce its incidence, methods for early diagnosis and a better understanding of the molecular mechanisms responsible for gastric cancer development and progression are required.

The development of gastric cancer, as with other types of cancer, is caused by risk factor-induced genetic alterations, such as the activation of oncogenes and the silencing of tumor suppressor genes (4-6). For example, H. pylori infection plays two major roles in gastric cancer development: it induces inflammation of the gastric mucosa and alters gene expression via the induction of mutations and DNA methylation (7). Indeed, $H$. pylori infection promotes the methylation and silencing of trefoil factor family 2 (TFF2), leading to gastric cancer development in humans (8). The TFF family includes secreted proteins characterized by a triple loop structure and the trefoil domain, and they are expressed in the gut (8-10). TFF1 is secreted by surface mucous and the pit epithelium in the fundus and antrum, whereas TFF2 is restricted to the fundic mucous neck cells, antrum and Brunner's glands, and TFF3 is found in intestinal cells (5). A previous study demonstrated that TFF1 is a stomachspecific tumor suppressor gene; however, the role of TFF2 in gastric cancer progression is less well understood (5). Recently, TFF2 was shown to play a protective role in the digestive tract (11); however, other studies have indicated that it is related to gastric diseases. For example, TFF2 expression has been shown to rapidly increase in gastrointestinal ulcerative diseases, particularly in the regenerating epithelium (12) or following non-steroidal anti-inflammatory drug treatment (13). Another study demonstrated that the level of TFF2 was markedly lower in the serum and tumor tissues of gastric cancer patients than in normal tissues, and this may be due to methylation of the TFF2 promoter $(14,15)$. However, it is not clear that TFF2 func- 
tions as a tumor suppressor in gastric cancer development. In a preliminary yeast two-hybrid screen, we previously found that the transcription factor, $\mathrm{Sp} 3$, is a candidate protein that binds to and potentially mediates the effects of TFF2 in gastric cancer cells (16). Thus, in this study, we characterized the interaction between TFF2 and Sp3 in the regulation of gastric cancer cell viability, apoptosis and invasion capacity.

\section{Materials and methods}

Cell lines and culture conditions. The normal human gastric mucosal cell line, GES-1, the gastric cancer cell line, BGC-823, and 293 cells were obtained from the Life Science College of Xiamen University (Xiamen, China) and maintained in Dulbecco's modified Eagle's medium (DMEM; Gibco, Rockville, MD, USA) supplemented with $10 \%$ fetal bovine serum (FBS) and $100 \mathrm{U} / \mathrm{ml}$ penicillin-streptomycin (both from Invitrogen, Carlsbad, CA, USA) at $37^{\circ} \mathrm{C}$ in a humidified incubator containing $5 \% \mathrm{CO}_{2}$.

Immunofluorescent detection of protein distribution. The gastric cancer cells were seeded onto coverslips in 6-well plates and grown for $24 \mathrm{~h}$. The cells were then fixed with freshly prepared $4 \%$ paraformaldehyde solution for $30 \mathrm{~min}$ on ice. After washing with phosphate-buffered saline (PBS) containing $0.1 \%$ Triton X-100, the cells were incubated overnight with antiTFF2 (orb214658; Biorbyt LLC, Berkeley, CA, USA) and/or anti-Sp3 (D20; Santa Cruz Biotechnology, Santa Cruz, CA, USA) antibodies at a dilution of 1:200. The following day, the cells were washed 3 times with PBS and then further incubated with fluorescein isothiocyanate (FITC)-conjugated goat antirabbit antibody (sc-3839; Santa Cruz Biotechnology) and/or Texas Red-conjugated goat anti-mouse antibody (1:600; T-862; Jackson ImmunoResearch Laboratories, West Grove, PA, USA). The cell nuclei were counterstained with 4',6-diamidino-2-phenylindole (DAPI; Gibco). The stained cells were viewed and scored under a BMX-60 microscope (Olympus, Tokyo, Japan) equipped with a cooled charge-coupled device and sensored camera (Cooke, Auburn Hills, MI, USA) and SlideBook software (Intelligent Imaging Innovations, Denver, CO, USA). At least 500 cells in each condition were reviewed and scored to calculate TFF2 and Sp3 positivity, and each experiment was repeated 3 times.

Construction of TFF2-containing plasmids and gene transfection. The GES-1 normal human gastric mucosal cells were grown, and total cellular RNA was isolated using TRIzol ${ }^{\circledR}$ reagent (Invitrogen) and reverse-transcribed into cDNA according to the manufacturer's instructions. To amplify TFF2, the primers used were 5'-AGAGAATTCGGATCCA TGGGACGGCGAGACG-3' (forward) and 5'-TGGCTCGA GCCCGGGGTAATGGCAGTCTTCCACAGAC-3' (reverse). PCR amplification was performed using primer enzyme (Fermentas, Vilnius, Lithuania) for 30 cycles of $94^{\circ} \mathrm{C}$ for $30 \mathrm{sec}, 58^{\circ} \mathrm{C}$ for $1 \mathrm{~min}$, and $72^{\circ} \mathrm{C}$ for $30 \mathrm{sec}$. The PCR products were then separated on $1.2 \%$ agarose gels, and TFF2 cDNA was recovered using a DNA Gel Extraction kit (Tiangene, Beijing, China). The recovered TFF2 cDNA was then cloned into the pcDNA6.0/HA-tag vector (Invitrogen) at the BamHI and XhoI sites. Following amplification, the plasmid was
DNA-sequenced for correct vector construction by Shanghai Majorbio Bio-Pharm Technology Co., Ltd. (Shanghai, China). The vector-only and pcDNA6.0/HA-tag/TFF2 plasmids were transfected into gastric cancer cells using Lipofectamine 2000 (Invitrogen) according to the manufacturer's instructions. We also expressed exogenous TFF 2 in the 293 cells by pcDNA6.0/ HA-tag/TFF2 transfection.

Construction of the Sp3 small hairpin RNA (shRNA) vector and gene transfection. To knock down Sp3 expression, a pU6 expression vector carrying shRNA targeting Sp3 (Sp3 shRNA) was constructed according to the methods described in previous studies $(17,18)$. The shRNA sense and antisense sequences targeting Sp3 cDNA were designed and synthesized by Invitrogen and inserted into the pU6 vector. Two different sets of Sp3 shRNA sequences were selected according to a previous study (19), i.e., Sp3 shRNA 1 (forward, 5'-CACCGG TGGGGCTTTCATTTCAAACGTGTGCTGTCCGTTTGAA GTGAAGGCTCCACCTTTTT-3' and reverse, 5'-GCATAA AAAGGTGGAGCCTTCACTTCAAACGGACAGCACACG TTTGAAATGAAAGCCCCACC-3') and Sp3 shRNA 2 (forward, 5'-CACCGGTGGGAGAGGTATCGATTACGT GTGCTGTCCGTAATTGGTACCTCTTCCACCTTTTT-3' and reverse, 5'-GCATAAAAAGGTGGAAGAGGTACCAA TTACGGACAGCACACGTAATCGATACCTCTCCCACC-3'). Following amplification, these plasmids were sequenced to confirm the shRNA construction. Following amplification, these plasmids were sequenced to confirm shRNA construction. shRNA1 was found to be more effective (data not shown). In the following paragraphs, $\mathrm{Sp} 3$ shRNA refers to $\mathrm{Sp} 3$ shRNA1 These vectors and the negative control pU6 vector were then transfected into the gastric cancer cells using Lipofectamine 2000 (Invitrogen) according to the manufacturer's instructions.

Protein extraction, immunoprecipitation and western blot analysis. Total cell lysates were harvested from $5 \times 10^{6}$ cells using $300 \mu \mathrm{l}$ of lysis buffer containing complete protease inhibitor cocktail (Roche, Basel, Switzerland) by rotation at $4^{\circ} \mathrm{C}$ for $30 \mathrm{~min}$ and then centrifuged at $12,000 \mathrm{x}$ g for $20 \mathrm{~min}$. The supernatant was collected, and the protein concentration was measured using the protein assay kit (Bio-Rad Laboratories, Hercules, CA, USA). For western blot analysis, an equal volume of cell lysate was denatured in sodium dodecyl sulfate (SDS) sample buffer, separated on a 10-12\% SDS-polyacrylamide gel with electrophoresis solution, and then transferred onto a polyvinylidene difluoride membrane (Bio-Rad Laboratories). The membranes were blocked in PBS-T (PBS containing $0.1 \%$ Tween-20) and $5 \%$ bovine serum albumin (Difco, BectonDickinson, Franklin Lakes, NJ, USA) at room temperature for $1 \mathrm{~h}$ and incubated with specific primary antibodies [antiTFF2 (orb214658; Biorbyt LLC) and anti-Sp3 (D20; Santa Cruz Biotechnology)] in PBS-T at $4^{\circ} \mathrm{C}$ for $2 \mathrm{~h}$. Following 3 washes with PBS-T, the membranes were incubated with horseradish peroxidase-conjugated goat anti-mouse secondary antibody (62-6520) or horseradish peroxidase-conjugated goat anti-rabbit secondary antibody (81-6120) (both from Zymed, San Diego, CA, USA) in PBS-T at room temperature for $1 \mathrm{~h}$. The membranes were then washed 3 times with PBS-T, and the protein level was detected using the ECL detection system (Sigma Biosciences, Santa Clara, CA, USA). 
For immunoprecipitation and western blot analysis, lysates of GES-1 cells expressing both TFF2 and Sp3 proteins were incubated with glutathione-sepharose beads and immunoprecipitated with an anti-Sp3 antibody. The precipitates were subjected to western blot analysis using anti-TFF2 and anti-Sp3 antibodies. The cell lysate incubated with antibody-free A/G-agarose served as a negative control.

Bromodeoxyuridine (BrdU) incorporation assay. To detect cell proliferation, we utilized the BrdU cell proliferation kit (Roche Diagnostics, Indianapolis, IN, USA). Briefly, the BGC-823 human gastric cancer cells were grown in 96-well plates and transfected with TFF2, siSp3, or control vectors for 24-48 h. Subsequently, $10 \mu \mathrm{l} / \mathrm{ml}$ BrdU was added to the cell cultures, and followed by further incubation at $37^{\circ} \mathrm{C}$ for 12 or $24 \mathrm{~h}$. The medium was then removed, and the cells were fixed with fresh $4 \%$ paraformaldehyde solution for $30 \mathrm{~min}$ at room temperature. To detect BrdU levels in the cells, an anti-BrdU antibody was added to each well followed by incubation for $90 \mathrm{~min}$ at room temperature. Following 3 washes with PBS-T, the cells were further incubated with the substrate solution for 30 min to develop color. After the addition of $\mathrm{H}_{2} \mathrm{SO}_{4}$, the absorbance of the cells at $450 \mathrm{~nm}$ was measured using a spectrophotometer [Eppendorf BioPhotometer D30, Eppendorf Biotechnology International Trade (Shanghai) Co., Ltd.].

Flow cytometric analysis. To analyze changes in cell apoptosis, the BGC-823 cells were grown in 96-well plates and transfected with TFF2, siSp3, or control vectors for 24-48 h with complete medium containing $7.5 \mathrm{mg} / \mathrm{ml}$ cisplatin. The cells were pelleted and resuspended in $0.3 \mathrm{ml}$ of PBS containing $3 \%$ calf serum. The cell pellets were then fixed by the dropwise addition of $0.7 \mathrm{ml}$ of methanol during gentle vortexing and stored overnight at $-20^{\circ} \mathrm{C}$. The following day, the fixed cells were pelleted, washed twice in PBS containing 3\% calf serum, and stained with Annexin V (Dojindo, Kumamoto, Japan) containing $100 \mu \mathrm{g} / \mathrm{ml}$ ribonuclease A according to the instructions provided with the kit and then with propidium iodide (PI, $50 \mu \mathrm{g} / \mathrm{ml}$ in PBS and 0.1\% Triton X-100; Dojindo) and protected from light until analysis. The cells were analyzed using a FACSCalibur flow cytometer (Becton-Dickinson).

Apoptosis antibody array. To determine the role of TFF2 and $\mathrm{Sp} 3$ binding in the regulation of gastric cancer cell apoptosis, the Proteome Profiler ${ }^{\mathrm{TM}}$ Human Apoptosis array kit (ARY009; R\&D Systems, Minneapolis, MN, USA) was used to detect the relative expression levels of 35 apoptosis-related proteins according to the manufacturer's instructions in BGC-823 cells showing differential expression of TFF2 compared to that in the control cells.

Tumor cell invasion assay. The BGC-823 cells were grown in 96-well plates and transfected with TFF2, siSp3, or control vectors for $24-48 \mathrm{~h}$, and then $1-5 \times 10^{4}$ cells without FBS were re-seeded in the top chamber of each insert (BD Biosciences, Franklin Lakes, NJ, USA) and the bottom chamber was filled with $20 \%$ FBS and incubated for $22 \mathrm{~h}$. At the end of the experiments, cells that migrated or invaded the surface of the filter were fixed with methanol for $30 \mathrm{~min}$ at $4^{\circ} \mathrm{C}$ and stained with $0.1 \%$ crystal violet containing $20 \%$ ethanol for $10 \mathrm{~min}$ at room temperature, and the total numbers of cells were enumerated under an IX71 inverted microscope (Olympus).

Statistical analysis. All statistical analyses were performed using Student's t-tests or one-way analysis of variance (ANOVA) implemented in GraphPad Prism 5.01 of GraphPad Software, Inc. (La Jolla, CA, USA) to evaluate differences between groups. A value of $\mathrm{P}<0.05$ was considered to indicate a statistially significant difference.

\section{Results}

Co-localization of TFF2 and Sp3 in GES-1 cells. Our previous study involving a yeast two-hybrid analysis revealed that TFF2 binds to Sp3, leading to antitumor activity in gastric cancer cells (16); thus, in this study, we first examined the co-localization of TFF2 and Sp3 in the normal gastric mucosal cell line, GES-1, using immunofluorescence. Our results revealed that the TFF2 protein was concentrated in the cytoplasm of GES-1 cells, whereas Sp3 was distributed in both the nuclei and cytoplasm (Fig. 1A). The proteins overlapped in the cytoplasm of the GES-1 cells.

Subsequently, using immunoprecipitation and western blot analysis, we confirmed that endogenous TFF2 binds to Sp3 protein in GES-1 cells (Fig. 1B). We also expressed exogenous TFF2 in the 293 cells by pcDNA6.0/HA-tag/TFF2 transfection (Fig. 1C). As shown by the results of western blot analysis, exogenous TFF2 was able to bind to Sp3 protein (Fig. 1D).

Effects of TFF2 expression on BGC-823 cell proliferation and apoptosis and the effect of Sp3 knockdown. We assessed the effects of TFF2 expression on the regulation of BGC-823 cell proliferation and apoptosis, and examined whether Sp3 knockdown alters the effects of TFF2 on gastric cancer cells. We constructed 4 strains of stably transfected BGC-823 cells: control cells, TFF2-overexpressing cells, cells in which Sp3 was knocked down, and TFF2-overexpressing cells in which Sp3 was also knocked down (namely, pcDNA6.0 + pU6, TFF2 + pU6, pcDNA6.0 + siSp3 and TFF2 + siSp 3 cells, respectively). The levels of TFF2 and Sp3 expression in the gastric cancer cells were examined by western blot analysis (Fig. 2A). We then assessed the changes in cell proliferation and apoptosis. The results of BrdU incorporation assay revealed that $\mathrm{Sp} 3$ promoted the proliferation of BGC-823 cells (pcDNA6.0 + pU6 vs. pcDNA6.0 + siSp3, Student's t-test, $\mathrm{P}<0.01)$, whereas TFF2 decreased cell proliferation (pcDNA6.0 + siSp3 vs. TFF2 + siSp3, Student's t-test, $\mathrm{P}<0.05$ ) (Fig. 2B). However, there was no significant effect on cell proliferation when both TFF2 and Sp3 (TFF2 + pU6) were overexpressed (Fig. 2B).

Finally, the data from the flow cytometric apoptosis assay indicated that TFF2 overexpression or $\mathrm{Sp} 3$ knockdown induced apoptosis (Fig. 2C). The BCG-823 cells were incubated with cisplatin for $36 \mathrm{~h}$, stained with Annexin V and PI, and analyzed by flow cytometry. We then compared the proportion of apoptotic cells between the pcDNA + pU6 (45.85\%), pU6 + TFF2 (51.03\%), siSp3 + pcDNA (38.12\%) and siSp3 + TFF2 (41.17\%) groups. The number of apoptotic cells was significantly higher in the gastric cancer cells with a high expression of Sp3 and with overexpression of TFF2 than in the cells with a high expression of Sp3 and a normal expres- 
A



DAPI

B

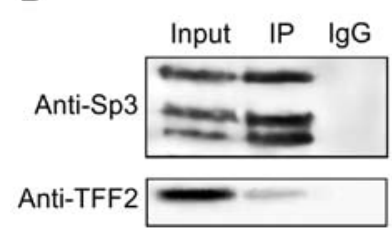

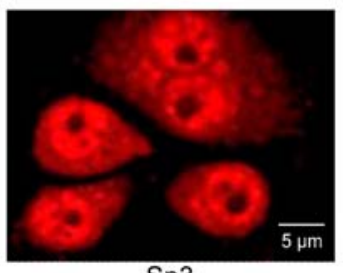

Sp3

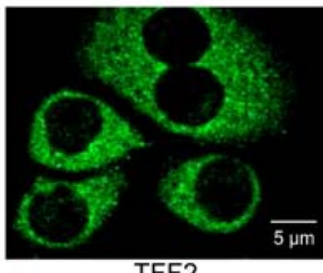

TFF2

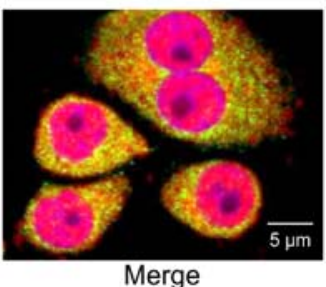

Merge c

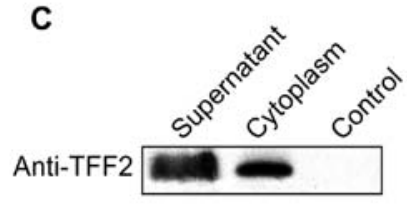

D

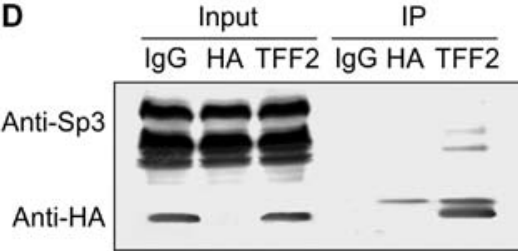

Figure 1. Interaction between trefoil factor family 2 (TFF2) and Sp3 in GES-1 cells. (A) Co-localization of endogenous TFF2 and Sp3 proteins in GES-1 cells examined by immunofluorescence. GES-1 cells were grown and fixed for the immunofluorescence analysis of TFF2 and Sp3 expression. The cells were first incubated with an anti-Sp3 antibody followed by Texas Red-conjugated goat anti-mouse antibody, and then with an anti-TFF2 antibody followed by fluorescein isothiocyanate (FITC)-conjugated goat anti-rabbit antibody. Images were recorded sequentially for DAPI, Sp3, and TFF2 and merged. TFF2 was mainly distributed in the cytoplasm. Sp3 was distributed in the cytoplasm and nucleus. The image shows that there was signal overlap for TFF2 and Sp3 in the cytoplasm. (B) Immunoprecipitation and western blot analysis. GES-1 cell lysates were first immunoprecipitated with an anti-Sp3 antibody or normal IgG as a control, and the precipitates were examined by western blot analysis with anti-Sp3 and anti-TFF2 antibodies. (C) Western blot analysis of exogenous HA-TFF2. 293 cells were grown and transfected with an HA-tag vector carrying TFF2 cDNA. Cell lysates were subjected to western blot analysis with anti-HA tag antibody. (D) Immunoprecipitation and western blot analysis were performed to analyze expression in 293 cells using an anti-HA-agarose antibody. The data from western blot analysis showed that exogenous TFF2 was able to bind to the Sp3 protein.

sion of TFF2 $(\mathrm{P}<0.05)$. After Sp3 was knocked down, gastric cancer cell apoptosis decreased significantly $(\mathrm{P}<0.05)$. With the combination of Sp3 knockdown and the overexpression of TFF2, the level of gastric cancer cell apoptosis did not decrease to a level significantly lower than that in the control cells. These results indicated that TFF2 and Sp3 alone significantly promoted the apoptosis of gastric cancer cells. TFF2 and Sp3 in combination further enhanced the apoptosis of gastric cancer cells. Accordingly, TFF2 and Sp3 had a synergistic effect on gastric cancer cell apoptosis.

Effects of TFF2 expression on BGC-823 cell invasion capacity and the effect of Sp3 knockdown. We assessed the effects of TFF2 expression on the regulation of BGC-823 cell invasion capacity and examined whether Sp3 knockdown alters the effects of TFF2 in gastric cancer cells. A tumor cell invasion assay was performed for the 4 BGC-823 cell sublines. Compared with the empty vector group, the gastric cancer cells with a high expression of Sp3 and overexpression of TFF2 did not exhibit a significant difference with respect to invasive ability (Student's t-test, $\mathrm{P}>0.05$ ). In the gastric cancer cells in which $\mathrm{Sp} 3$ was knocked down, the invasive ability was significantly lower than that of the control cells (pcDNA6.0 + pU6; Student's t-test, $\mathrm{P}<0.05$ ). In the gastric cancer cells in which $\mathrm{Sp} 3$ was knocked down and with TFF2 overexpression, the invasive ability was markedly greater (Fig. 2D). These results indicated that TFF2 and Sp3 alone significantly promoted the invasive ability of the gastric cancer cells. TFF2 and Sp3 together, did not further enhance the invasive ability of the gastric cancer cells. This suggests that TFF2 and Sp3 have an antagonistic effect on gastric cancer cell invasion.

Potential signaling pathway in TFF2-induced tumor cell apoptosis and interaction with Sp3. To confirm the TFF2-induced apoptotic effects and the interaction between TFF2 and Sp3 in gastric cancer cells, we used the Proteome Profiler ${ }^{\mathrm{TM}}$ Human Apoptosis array kit. We detected 14 apoptosis-related proteins that were differentially expressed in the TFF2-overexpressing gastric cancer cells compared to the pcDNA6.0 control cells (Fig. 3A and B). Using 4 different stable gastric cancer sublines, we found that the pro-apoptotic protein, Bid, was upregulated, but the pro-apoptotic protein, Bax, was downregulated; however, the anti-apoptotic proteins, Bcl-xL and Mcl-1, were downregulated in the TFF2-overexpressing cells (Fig. 3C). TFF2 expression also downregulated the protein expression of NF- $\kappa \mathrm{B}$ (Fig. 3D). However, following Sp3 knockdown, the effects of TFF2 on the expression of these proteins were reduced (Fig. 3C and D). These results demonstrated that TFF2 and Sp3 induced apoptotic effects via related signaling proteins. There was an antagonistic interaction between TFF2 and Sp3 with respect to cancer cell invasion. The high expression of TFF2 and Sp3 alone regulated the cell cycle in gastric cancer by blocking of the effect of GSK-3, p-NF- $\mathrm{B}, \mathrm{IKK} \alpha /$ IKK $\beta$, and other signaling pathway proteins. Thus, TFF2 and $\mathrm{Sp} 3$ have a greater effect in combination with respect to gastric cancer cell apoptosis.

\section{Discussion}

Increasing evidence has indicated that TFFs play critical roles in protecting the gastrointestinal tract from inflammation or tumorigenesis; they are involved in the repair of the gastrointestinal epithelium and the suppression of tumor formation in the stomach. By contrast, TFF2 deficiency promotes inflammation in gastric mucosae and is associated with gastric malignancy (15). However, few studies have examined its role at the cellular and molecular level, and the underlying molecular mechanisms that mediate its effects are unknown (20). Our 
A

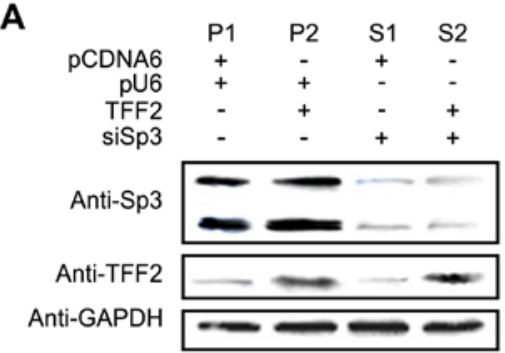

C

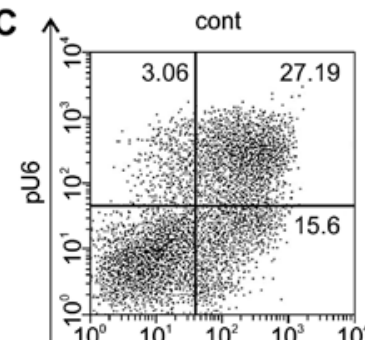

$\overline{\mathbf{2}}$
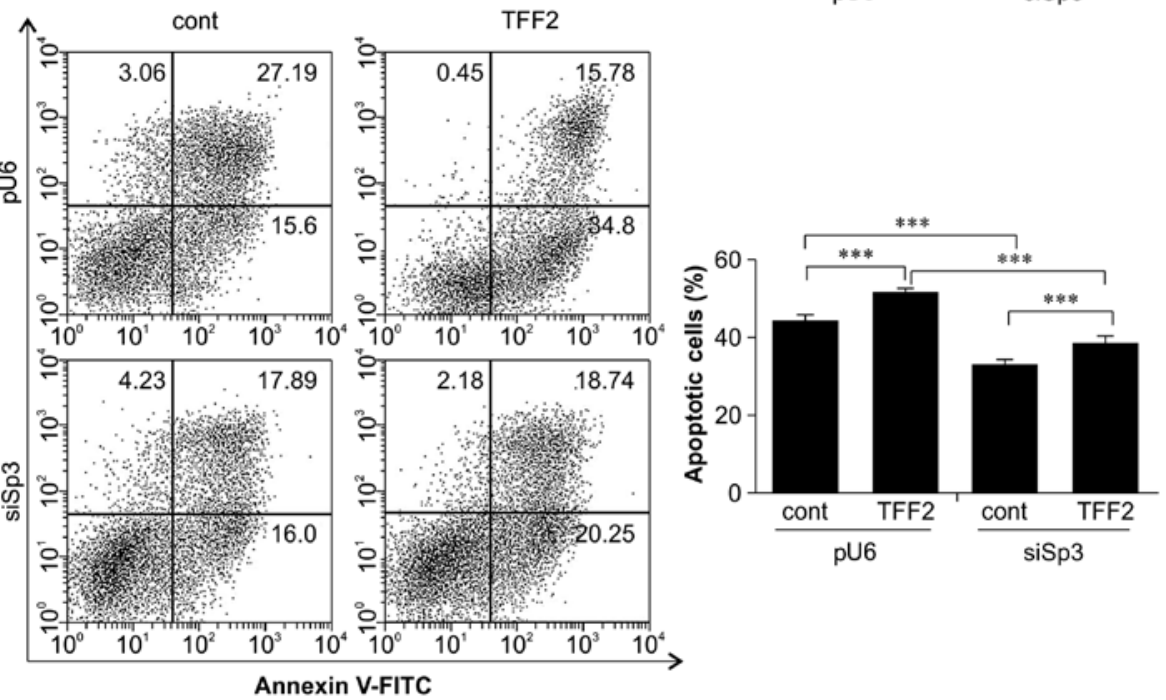

D
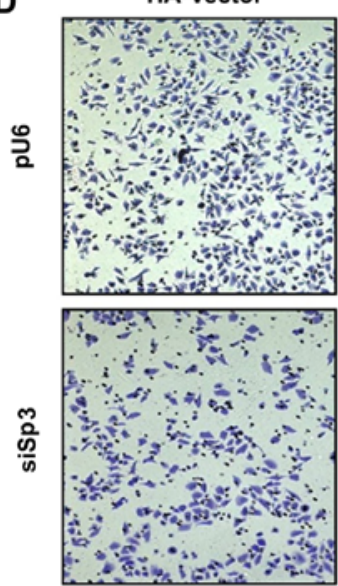

$\times 100$

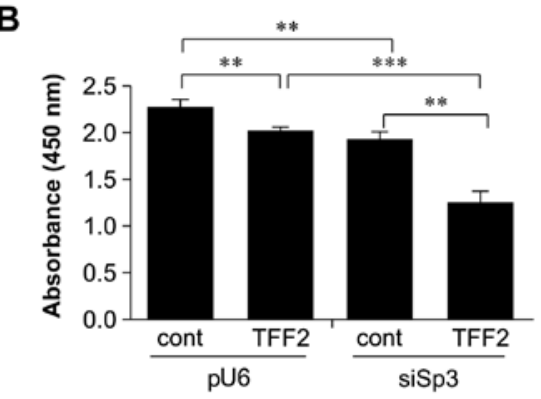

HA-TFF2
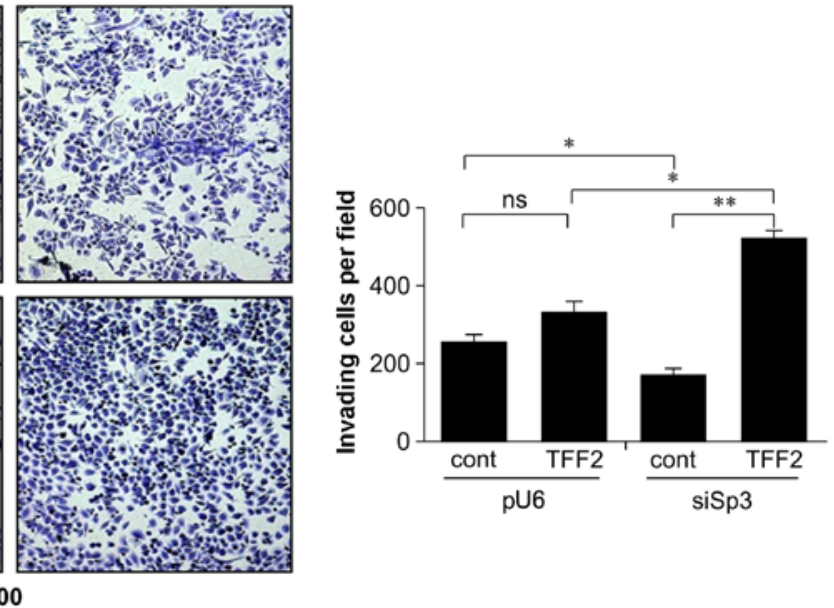

Figure 2. Effects of trefoil factor family 2 (TFF2) expression on gastric cancer cell proliferation and apoptosis and the effect of stable Sp3 knockdown in BGC-823 gastric cancer cells. (A) Western blot analysis of TFF2 and Sp3 expression in gastric cancer cells. BGC-823 cells were grown and stably transfected with HA-TFF2, pU6-siSp3, or control vectors, and cell lysates were subjected to western blot analysis for TFF2 and siSp3 expression. GAPDH was used as a loading control. (B) Bromodeoxyuridine (BrdU) incorporation assay. The 4 types of BGC-823 cell sublines were grown and incubated with BrdU for up to $22 \mathrm{~h}$ and then subjected to spectrophotometer analysis of optical absorbance levels at $450 \mathrm{~nm}$. Data are expressed as the means $(\mathrm{nm}) \pm \mathrm{SD}$ of 3 separate experiments and were analyzed by Student's t-tests. The BrdU incorporation assay showed that Sp3 promoted the proliferation of BGC-823 cells (pcDNA6.0 + pU6 vs. pcDNA6.0 + siSp3, P<0.01), whereas TFF2 reduced cell proliferation (pcDNA6.0 + siSp3 vs. TFF2 + siSp3, P<0.05). (C) Flow cytometric analysis of BCG-823 cells following incubation with cisplatin for $36 \mathrm{~h}$ and subsequent staining with Annexin $\mathrm{V}$ and propidium iodide (PI). Dots in Annexin $\mathrm{V}^{-} / \mathrm{PI}^{-}$, Annexin $\mathrm{V}^{+} / \mathrm{PI}^{-}$, and $\mathrm{Annexin}^{+} / \mathrm{PI}^{+}$indicate intact cells, cells undergoing early apoptosis and dead cells, respectively. Data are shown as the means $(\%) \pm \mathrm{SD}$ of 3 separate experiments and were analyzed by Student's t-tests. We compared the frequency of apoptotic cells for pcDNA + pU6 (45.85\%), pU6 + TFF2 (51.03\%), siSp3 + pcDNA (38.12\%), and siSp3 + TFF2 $(41.17 \%)$ cells. In cells with a high expression of Sp3 and overexpression of TFF2, gastric cancer cell apoptosis was significantly higher than that in the control cells $(\mathrm{P}<0.05)$. In Sp3 knockdown gastric cancer cells, apoptosis decreased significantly $(\mathrm{P}<0.05)$. In cells with Sp3 knockdown and overexpression of TFF2, the apoptosis level did not decrease obviously. These results indicated that TFF2 and Sp3 alone significantly promoted the apoptosis of gastric cancer cells. TFF2 and $\mathrm{Sp} 3$ in combination further enhanced the levels of gastric cancer cell apoptosis. Accordingly, TFF2 and Sp3 had a synergistic effect on gastric cancer cell apoptosis. (D) Tumor cell invasion assay for the four BGC-823 cell sublines. Data are shown as the means \pm SD of 3 separate experiments and were analyzed by Student's t-tests. Compared with the empty vector group, gastric cancer cells with a high expression of Sp3 and overexpression of TFF2 did not differ significantly with respect to invasive ability $(\mathrm{P}>0.05)$. In cells with $\mathrm{Sp} 3$ knockdown, the invasive ability decreased significantly $(\mathrm{P}<0.05)$. In gastric cancer cells with Sp3 knockdown and TFF2 overexpression, the invasive ability markedly increased. These results indicated that TFF2 and Sp3 alone significantly promoted the invasive ability of gastric cancer cells. The combination of TFF2 and Sp3 did not further enhance the invasive ability of gastric cancer cells. This suggested that TFF2 and Sp3 had an antagonistic effect on gastric cancer cell invasion. ns, not significant; ${ }^{* * *} \mathrm{P}<0.01,{ }^{* *} \mathrm{P}<0.05$, and ${ }^{*} \mathrm{P}<0.1$ compared to the control. Groups in the bar charts are as follows: pU6 cont (control), pcDNA6.0 + pU6; pU6 TFF2, TFF2 + pU6; siSp3 cont, pcDNA6.0 + siSp3; and siSp3 TFF2, TFF2 + siSp3. 
A
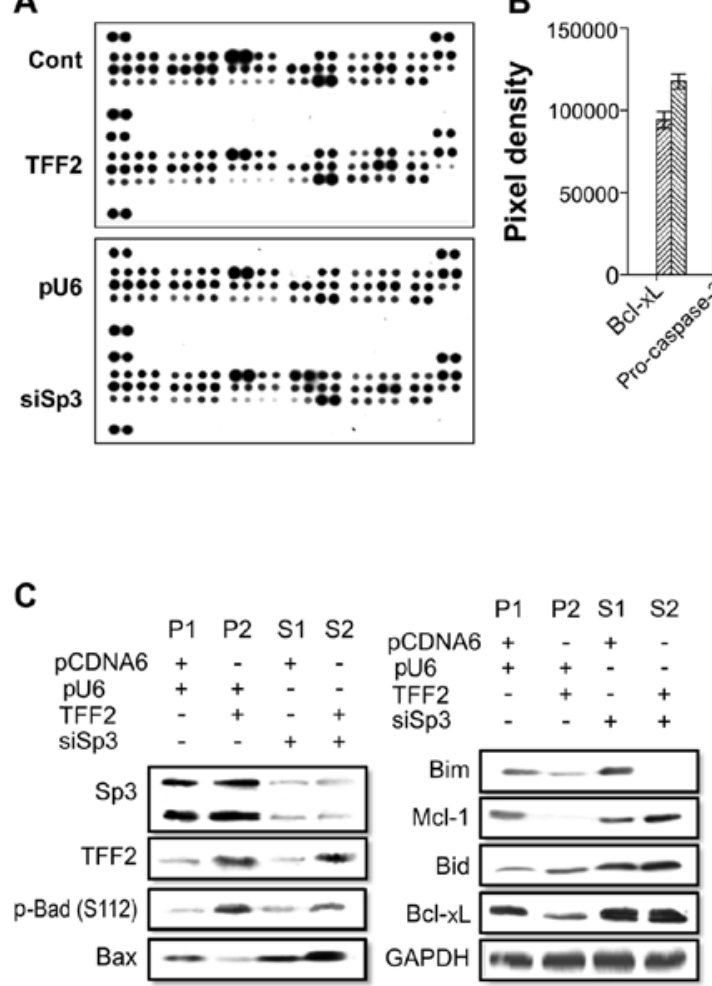

B
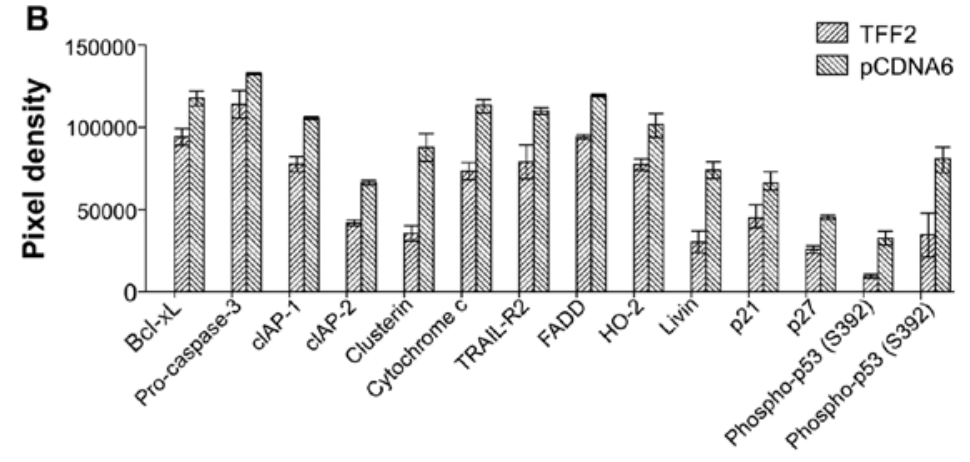

D

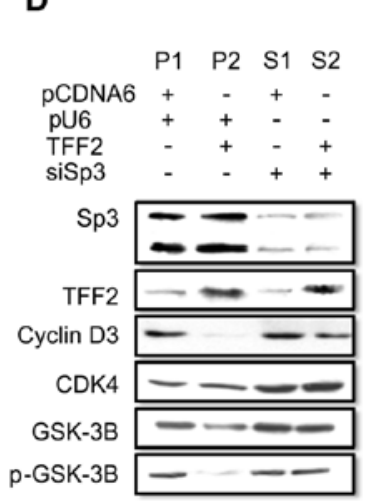

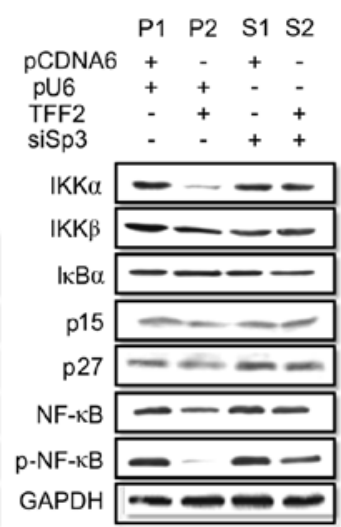

Figure 3. Effects of trefoil factor family 2 (TFF2) expression on the expression of apoptosis signaling pathway genes and the effect of Sp3 knockdown in BGC-823 cells. (A) Human apoptosis gene array analysis. Similar levels of apoptosis-related proteins were detected between cells overexpressing TFF2 and parental BGC-823 cell lines. (B) Quantitative analysis of the gene array shown in (A). Fourteen apoptosis-related proteins were significantly differentially expressed between cells overexpressing TFF2 and the control cells (pcDNA6.0 + pU6). (C) Western blot analysis of apoptotic protein expression in these 4 BGC823 cell sublines. (D) Western blot analysis of cell cycle signaling protein expression in the 4 BGC-823 cell sublines.

current study demonstrated that the transcription factor, $\mathrm{Sp} 3$, acts as a novel TFF2 binding partner in the GES-1 gastric mucosal cell line. Our data further suggested that TFF2 binds to the short isoform of the Sp3 protein, but not to the long isoform. Additionally, we showed that TFF2 binds to the cytoplasmic form of the Sp3 protein. However, the biological significance of this phenomenon remains to be determined. Indeed, the Sp3 protein is ubiquitously expressed in all mammalian cells (21). It belongs to the specificity protein/Krüppel-like factor (SP/KLF) transcription factor family containing a specific DNA-binding domain composed of a combination of three conserved $\mathrm{Cys}_{2} \mathrm{His}_{2}$ zinc fingers (22). Sp3 regulates the expression of a number of genes; consistent with this, our data demonstrated that TFF2 binds to Sp3 in the cytoplasm, resulting in the increased expression of genes involved in the suppression of cell proliferation and the induction of apoptosis (23). The Sp3 protein exists in multiple isoforms: two long fragments and two short ones (24-26). Previous research on Sp3 has focused on its DNA-binding sites and gene regulation (27). Thus, multiple promoters have known binding sites for $\mathrm{Sp} 3$, which is clearly important for gene expression regulation (28). However, the interaction between TFF2 and Sp3 has not been documented. In this study, to the best of our knowledge, we demonstrated for the first time that TFF2 interacts with Sp3 to mediate the biological functions of TFF2 in gastric cancer cells.

Furthermore, we assessed the biological function of the TFF2 protein in gastric cancer cells and clarified the role of Sp3 by establishing 4 stable cell lines with different expression levels for each locus. Using BrdU incorporation and flow cytometric assays, we found that TFF2 expression inhibited cell proliferation, but that it promoted the apoptosis of BGC-823 cells. Sp3 knockdown antagonized the effects of TFF2 on gastric cancer cells, indicating that the interaction between TFF2 and Sp3 is essential for TFF2 function in gastric cancer cells. Further studies are required to confirm this preliminary observation.

TFF2 and Sp3 both promote invasion in gastric cancer cells. However, the effect was less notable in cells overexpressing TFF2 and Sp3. A previous study demonstrated that despite its role as a tumor suppressor, TFF2 can induce gastric cancer cell invasion (29), although the mechanisms underlying this effect are unknown. We extended these observations by exploring the molecular mechanisms through which TFF2 and Sp3 interact to regulate tumor cell apoptosis. We identified 14 differentially expressed proteins in response to TFF2 expression in gastric cells. Our data were consistent with those of previous studies showing that the Bcl-2 family (30) and NF- $\mathrm{BB}$ signaling proteins $(31,32)$ are important in gastrointestinal tumorigenesis. TFF2 expression downregulated the expression of Bcl-xL and $\mathrm{NF}-\kappa \mathrm{B}$ family signal proteins in the 4 stable cell lines expressing different levels of TFF2 and Sp3. We also demonstrated that the expression of pro-apoptotic proteins (e.g., Bid) may be induced in response to TFF2 expression in gastric cancer cells. However, following Sp3 knockdown in these cells, the effects of TFF2 protein were significantly reduced, further indicating that $\mathrm{Sp} 3$ protein mediates TFF2 antitumor activity. 
In conclusion, in the present study, we demonstrated that endogenous TFF2 binds to Sp3 in GES-1 cells and that the effects of TFF2 antitumor activity (e.g., the inhibition of tumor proliferation and the induction of apoptosis) are mediated by this interaction. However, further studies are required to confirm the precise mechanisms through which TFF2 interacts with the Sp3 protein to mediate the effects of TFF2 in gastric cancer cells.

\section{Acknowledgements}

This study was supported in part by grant $3502 Z 20126015$ from the Science and Technology Innovation Foundation of Xiamen (Fujian, China). The authors are grateful to members of the Department of Gastroenterology, Zhongshan Hospital Xiamen University. We would also like to thank Ms. Diana Monteiro for assisting with the manuscript revision.

\section{References}

1. Jemal A, Bray F, Center MM, Ferlay J, Ward E and Forman D: Global cancer statistics. CA Cancer J Clin 61: 69-90, 2011.

2. Lozano R, Naghavi M, Foreman K, Lim S, Shibuya K, Aboyans V, Abraham J, Adair T, Aggarwal R, Ahn SY, et al: Global and regional mortality from 235 causes of death for 20 age groups in 1990 and 2010: A systematic analysis for the Global Burden of Disease Study 2010. Lancet 380: 2095-2128, 2012.

3. Bertuccio P, Chatenoud L, Levi F, Praud D, Ferlay J, Negri E, Malvezzi $\mathrm{M}$ and La Vecchia C: Recent patterns in gastric cancer: A global overview. Int J Cancer 125: 666-673, 2009.

4. Ganapathy S, Sengupta S, Wawrzyniak PK, Huber V, Buda F, Baumeister U, Würthner F and de Groot HJ: Zinc chlorins for artificial light-harvesting self-assemble into antiparallel stacks forming a microcrystalline solid-state material. Proc Natl Acad Sci USA 106: 11472-11477, 2009.

5. Jiang P, Yu G, Zhang Y, Xiang Y,Zhu Z, Feng W, Lee W and Zhang Y: Promoter hypermethylation and downregulation of trefoil factor 2 in human gastric cancer. Oncol Lett 7: 1525-1531, 2014.

6. Yu G, Jiang P, Xiang Y, Zhang Y, Zhu Z, Zhang C, Lee S, Lee $\mathrm{W}$ and $Z$ hang $Y$ : Increased expression of protease-activated receptor 4 and trefoil factor 2 in human colorectal cancer. PLoS One 10: e0122678, 2015.

7. Chiba T, Marusawa H, Seno $\mathrm{H}$ and Watanabe N: Mechanism for gastric cancer development by Helicobacter pylori infection. J Gastroenterol Hepatol 23: 1175-1181, 2008.

8. Xu Q, Chen MY, He CY, Sun LP and Yuan Y: Promoter polymorphisms in trefoil factor 2 and trefoil factor 3 genes and susceptibility to gastric cancer and atrophic gastritis among Chinese population. Gene 529: 104-112, 2013.

9. Tsai YC, Hsiao WH, Lin SH, Yang HB, Cheng HC, Chang WL, Lu CC and Sheu BS: Genomic single nucleotide polymorphisms in the offspring of gastric cancer patients predispose to spasmolytic polypeptide-expressing metaplasia after $H$. pylori infection. J Biomed Sci 22: 16, 2015.

10. Belovari T, Bijelić N, Tolušić Levak M and Baus Lončar M Trefoil factor family peptides TFF1 and TFF3 in the nervous tissues of developing mouse embryo. Bosn J Basic Med Sci 15: 33-37, 2015.

11. Hanisch FG, Bonar D, Schloerer N and Schroten H: Human trefoil factor 2 is a lectin that binds $\alpha$-GlcNAc-capped mucin glycans with antibiotic activity against Helicobacter pylori. J Biol Chem 289: 27363-27375, 2014.

12. Fujimoto K, Fujii G, Taguchi K, Yasuda K, Matsuo Y,Hashiyama A, Mutoh M, Tanaka $\mathrm{H}$ and Wada M: Involvement of trefoil factor family 2 in the enlargement of intestinal tumors in Apc(Min/+) mice. Biochem Biophys Res Commun 463: 859-863, 2015.

13. Ortiz-Masiá D, Hernández C, Quintana E, Velázquez M, Cebrián S, Riaño A, Calatayud S, Esplugues JV and Barrachina MD: iNOS-derived nitric oxide mediates the increase in TFF2 expression associated with gastric damage: Role of HIF-1. FASEB J 24: 136-145, 2010.

14. Aikou S, Ohmoto Y, Gunji T, Matsuhashi N, Ohtsu H, Miura H, Kubota K, Yamagata Y, Seto Y, Nakajima A, et al: Tests for serum levels of trefoil factor family proteins can improve gastric cancer screening. Gastroenterology 141: 837-845.e1, 7, 2011.
15. Peterson AJ, Menheniott TR, O'Connor L, Walduck AK, Fox JG, Kawakami K, Minamoto T, Ong EK, Wang TC, Judd LM, et al: Helicobacter pylori infection promotes methylation and silencing of trefoil factor 2, leading to gastric tumor development in mice and humans. Gastroenterology 139: 2005-2017, 2010.

16. Zhan XJ, Ren JL, Xu HZ, Dong J, Zhou F, Zhou F, Pan JS and Xiao HM: Identification of genes encoding human trefoil factor 2-interacting proteins by screening a cDNA library of gastric cancer cells. World Chin J Dig Chin 17: 2767-2772, 2009

17. Miyagishi M and Taira K: U6 promoter-driven siRNAs with four uridine 3 ' overhangs efficiently suppress targeted gene expression in mammalian cells. Nat Biotechnol 20: 497-500, 2002.

18. Guleng B, Tateishi K, Ohta M, Kanai F, Jazag A, Ijichi H, Tanaka Y, Washida M, Morikane K, Fukushima Y, et al: Blockade of the stromal cell-derived factor-1/CXCR4 axis attenuates in vivo tumor growth by inhibiting angiogenesis in a vascular endothelial growth factor-independent manner. Cancer Res 65: 5864-5871, 2005.

19. Jazag A, Ijichi H, Kanai F, Imamura T, Guleng B, Ohta M, Imamura J, Tanaka Y, Tateishi K, Ikenoue T, et al: Smad4 silencing in pancreatic cancer cell lines using stable RNA interference and gene expression profiles induced by transforming growth factor-beta. Oncogene 24: 662-671, 2005.

20. Kim HJ, Kim JC, Min JS, Kim MJ, Kim JA, Kor MH, Yoo HS and Ahn JK: Aqueous extract of Tribulus terrestris Linn induces cell growth arrest and apoptosis by down-regulating NF- $\mathrm{kB}$ signaling in liver cancer cells. J Ethnopharmacol 136: 197-203, 2011.

21. Meinders M, Kulu DI, van de Werken HJ, Hoogenboezem M, Janssen H, Brouwer RW, van Ijcken WF, Rijkers EJ, Demmers JA, Krüger I, et al: $\mathrm{Sp} 1 / \mathrm{Sp} 3$ transcription factors regulate hallmarks of megakaryocyte maturation and platelet formation and function. Blood 125: 1957-1967, 2015.

22. Hertel J, Hirche C, Wissmann C, Ebert MP and Höcker M: Transcription of the vascular endothelial growth factor receptor-3 (VEGFR3) gene is regulated by the zinc finger proteins Sp1 and $\mathrm{Sp} 3$ and is under epigenetic control: Transcription of vascular endothelial growth factor receptor 3. Cell Oncol (Dordr) 37: 131-145, 2014.

23. Davie JR, He S, Li L, Sekhavat A, Espino P, Drobic B, Dunn KL, Sun JM, Chen HY, Yu J, et al: Nuclear organization and chromatin dynamics - Sp1, Sp3 and histone deacetylases. Adv Enzyme Regul 48: 189-208, 2008.

24. Katsuyama M, Hirai H, Iwata K, Ibi M, Matsuno K, Matsumoto M and Yabe-Nishimura $\mathrm{C}: \mathrm{Sp} 3$ transcription factor is crucial for transcriptional activation of the human NOX4 gene. FEBS J 278: 964-972, 2011

25. Hoffmann C, Zimmermann A, Hinney A, Volckmar AL, Jarrett HW, Fromme T and Klingenspor M: A novel SP1/Sp3 dependent intronic enhancer governing transcription of the UCP3 gene in brown adipocytes. PLoS One 8: e83426, 2013.

26. Lian S, Potula HH, Pillai MR, Van Stry M, Koyanagi M, Chung L, Watanabe M and Bix M: Transcriptional activation of Mina by Sp1/3 factors. PLoS One 8: e80638, 2013.

27. Zelko IN, Mueller MR and Folz RJ: Transcription factors sp1 and $\mathrm{sp} 3$ regulate expression of human extracellular superoxide dismutase in lung fibroblasts. Am J Respir Cell Mol Biol 39: 243-251, 2008.

28. Wilson AJ, Chueh AC, Tögel L, Corner GA, Ahmed N, Goel S, Byun DS, Nasser S, Houston MA, Jhawer M, et al: Apoptotic sensitivity of colon cancer cells to histone deacetylase inhibitors is mediated by an $\mathrm{Sp} 1 / \mathrm{Sp} 3$-activated transcriptional program involving immediate-early gene induction. Cancer Res 70 : 609-620, 2010.

29. Tu SP, Chi AL, Ai W, Takaishi S, Dubeykovskaya Z, Quante M, Fox JG and Wang TC: p53 inhibition of AP1-dependent TFF2 expression induces apoptosis and inhibits cell migration in gastric cancer cells. Am J Physiol Gastrointest Liver Physiol 297: G385-G396, 2009.

30. Hiraki M, Kitajima Y, Sato S, Nakamura J, Hashiguchi K, Noshiro H and Miyazaki K: Aberrant gene methylation in the peritoneal fluid is a risk factor predicting peritoneal recurrence in gastric cancer. World J Gastroenterol 16: 330-338, 2010.

31. Auyeung KK, Law PC and Ko JK: Astragalus saponins induce apoptosis via an ERK-independent $\mathrm{NF}-\kappa \mathrm{B}$ signaling pathway in the human hepatocellular HepG2 cell line. Int J Mol Med 23: 189-196, 2009.

32. Jani TS, DeVecchio J, Mazumdar T, Agyeman A and Houghton JA: Inhibition of NF-kappaB signaling by quinacrine is cytotoxic to human colon carcinoma cell lines and is synergistic in combination with tumor necrosis factor-related apoptosis-inducing ligand (TRAIL) or oxaliplatin. J Biol Chem 285: 19162-19172, 2010. 